\title{
Proteomic pilot study of tuberculosis pleural effusion
}

\author{
Jiaqiang Zhang, Li-Zhou Fang, Ling Liu, Jianqing Zhang, Weiping Fu and Luming Dai ${ }^{*}$ \\ The Second Department of Respiratory Medicine, The First Affiliated Hospital of Kunming Medical \\ University, 295 \#, Xichang Road, Xishan District, Kunming City, Yunnan Province, China
}

\begin{abstract}
To discuss the differences in protein expression among tuberculosis pleural effusion (TBPE), malignant pleural effusion (MPE) and transudative pleural effusion (TSPE). We recruited 50 patients with pleural effusion, including 20 TBPEs, 17 MPEs and 13 TSPEs. Using the two-dimensional electrophoresis (2-DE) and matrix-assisted laser desorption ionization-time of flight-mass spectrometry (MALDI-TOF-MS), we acquired the peptide mass finger printings (PMFs) of featured proteins. Then, we identified characteristic proteins by searching in the protein databank of the National Center of Biotechnology Information (NCBI) and assessed their diagnostic significance. We found five characteristic proteins: C1-inhibitor (C1-INH), transthyretin (TTR), human complement fragment $3 \mathrm{~b}(\mathrm{C} 3 \mathrm{~b})$, human ceruloplasmin (CP), and Z34c protein fragment $\mathrm{Fc}(\mathrm{Z} 34 \mathrm{c}-\mathrm{Fc})$. C1-INH shows a high expression in TBPE and a low expression in MPE while TTR and C3 show low expression in TBPE and high expressions in MPE. Z34c-Fc and CP have a higher expression in TBPE than in TSPE. No common characteristic protein was found between MPE and TSPE. Statistic analysis consisted of paired t-tests and the difference between them is significance $(\mathrm{P}<0.05)$. C1-inhibitor $(\mathrm{C} 1-\mathrm{INH})$, transthyretin $(\mathrm{TTR})$, human Complement fragment $3 \mathrm{~b}(\mathrm{C} 3 \mathrm{~b})$, human Ceruloplasmin $(\mathrm{CP})$ and $\mathrm{Z} 34 \mathrm{c}$ protein fragment $\mathrm{Fc}(\mathrm{Z} 34 \mathrm{c}-\mathrm{Fc})$ may provide an additional perspective in the differential diagnosis of PE.
\end{abstract}

Keywords: Tuberculosis pleural effusion, proteomic, 2-DE; MALDI-TOF-MS

\section{Introduction}

Tuberculosis (TB) causes poor health among millions of people each year and ranks as the second leading cause of death from an infectious disease worldwide, after the human immunodeficiency virus (HIV) [1]. Moreover, $82 \%$ of the global TB burden was concentrated in 22 low-income countries with few financial resources and weak healthcare infrastructure [2]. TBPE is the second most common form of extra-pulmonary TB, behind only lymph node TB. The diagnosis of a TBPE requires either a positive culture from pleural fluid or pleural tissue or the presence of granulomas in the pleura [3]. Although several methods including pleural biopsy, ELISPOT Assay, and Xpert MTB/RIF Assay have gained wide acceptance in the diagnosis of TBPE, the use of these investigative tools may be limited by availability and quality control, especially in developing nations [4]. Therefore, it remains an urgent challenge to ensure that new diagnostic tests are suitable for use in peripheral health facilities in

\footnotetext{
* Address for correspondence: Luming Dai, The Second Department of Respiratory Medicine, The First Affiliated Hospital of Kunming Medical University, 295 \#, Xichang Road, Xishan District, Kunming City, Yunnan Province, China. Tel.: +8613577096065; Fax: 86-0871-65336015; E-mail: dailuming6622@hotmail.com.
} 
resource-poor countries [5]. Pleural effusion in general contains a high protein concentration, but the variability in protein composition among different pleural effusions is still not well understood. The aims of this study are to identify the differential expression of major proteins in TBPE, MPE and TSPE and to use this information to propose a rapid and accurate diagnostic method for clinical use.

\section{Material and methods}

\subsection{Patients}

Study participants consisted of 50 inpatients with pleural effusions admitted to the department of respiratory medicine of the first affiliated hospital of Kunming Medical University from May 2010 to June 2011. Among these patients, 20 TBPEs were diagnosed by thoracoscopic pathology results or the detection of mycobacterium tuberculosis in a brush biopsy or pleura biopsy; these 20 cases, proved to be anti-tuberculosis effective and with no indications of MPE. Additionally, 17 MPEs were diagnosed by thoracoscopic pathology results or lymph node biopsy, with no indications of TBPEs. The final 13 patients were diagnosed with TSPEs in conjunction with heart failure, hepatocirrhosis or hypoproteinemia, with no indications of TBPE or MPE. Pleural fluid samples (5 ML) were collected from each patient and kept in a $-80^{\circ} \mathrm{C}$ refrigerator for analysis.

The ethics committee of Kunming Medical University approved this study and all aspects of the study were conducted under the committee's supervision. All patients involved in the study were fully informed in advance.

\subsection{Methods}

The samples were mixed and concentrated in an ultrafilter. Isoelectric focusing after joining the lysis buffer allowed us to produce several gels. We balanced the gels and ran the sodium dodecyl sulfate polyacrylamide gel electrophoresis (SDS-PAGE); after electrophoresis, we dyed and decolored the gels. We placed them on the plate of a gel image scanner and obtained the images displayed in Figures 1-3. All the images were analyzed by Image Master 2D Platinum (Amasya). The resolution was set at $300 \mathrm{dpi}$, and the size proportion of the images and gels was 1:1. The images were saved as TIF files. After angel modification, noise filtering and other corrections, the images were sent to the Image Master software for further analysis. After background deduction and image correction, we

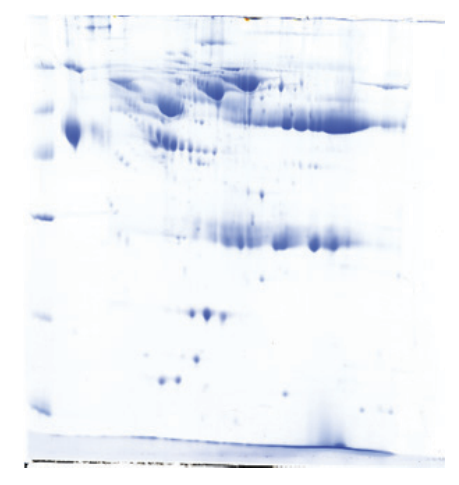

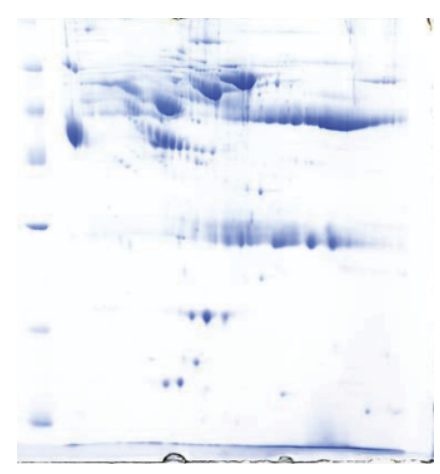

Fig. 1. Gel images of TBPE.

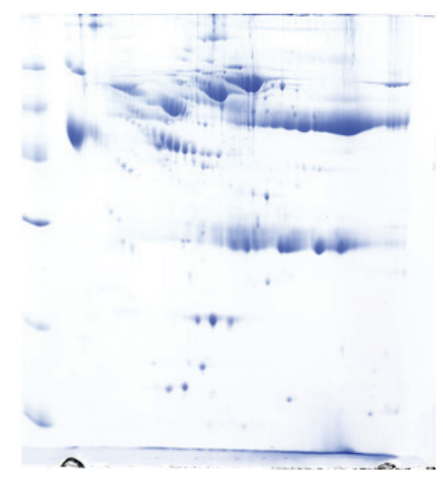



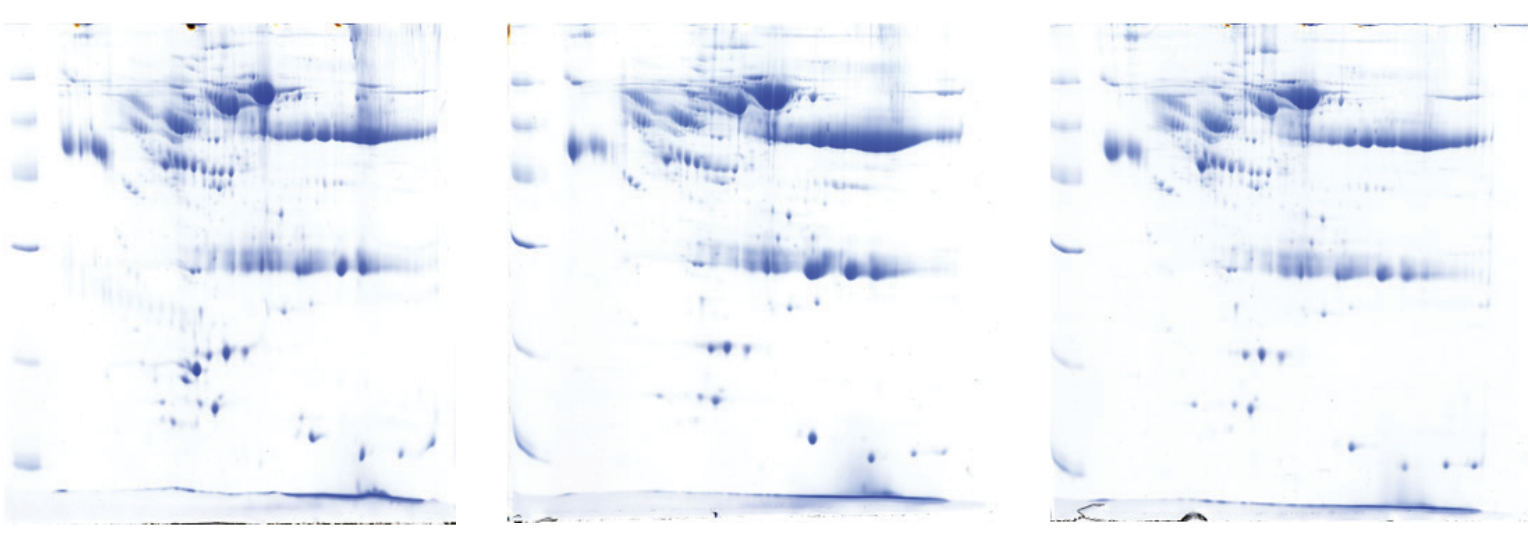

Fig. 2. Gel images of MPE.
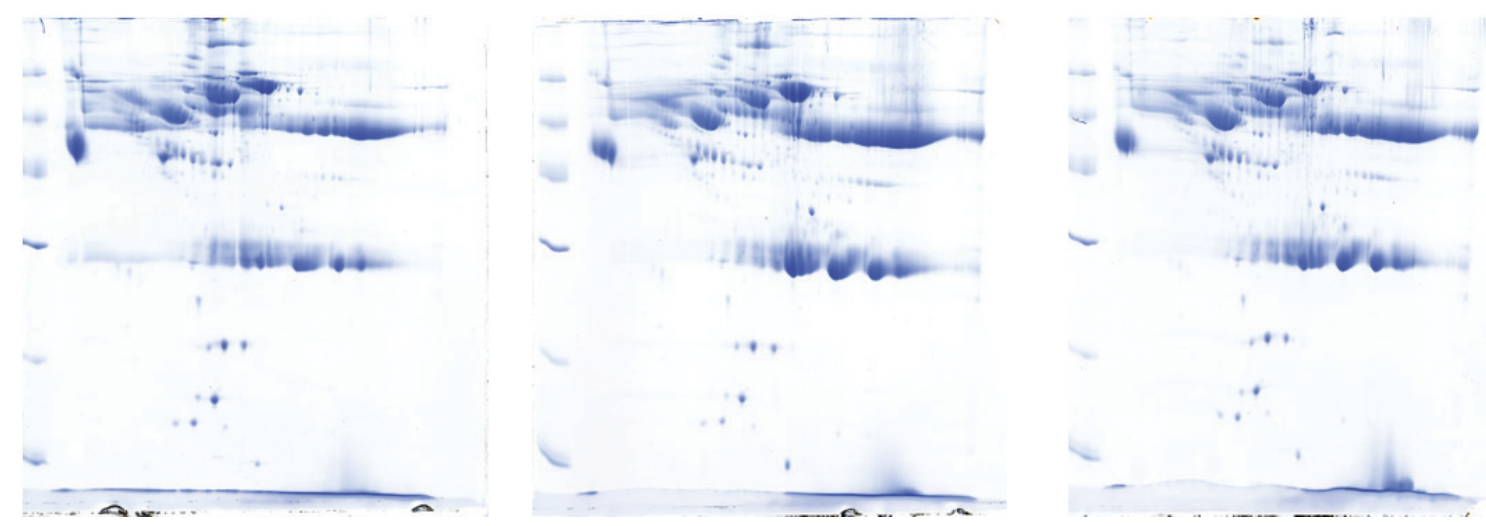

Fig. 3. Gel images of TSPE.

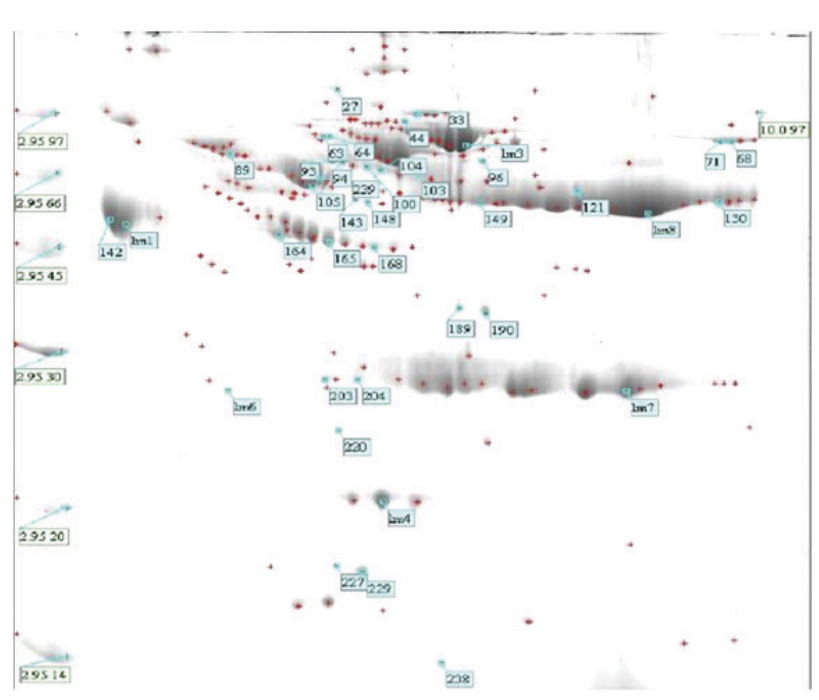

Fig. 4. The 2-DE map of samples in the TBPE group. Note: $\mathrm{pH}$ range from 3 to 10 ; molecular weight from 14-97 kD.

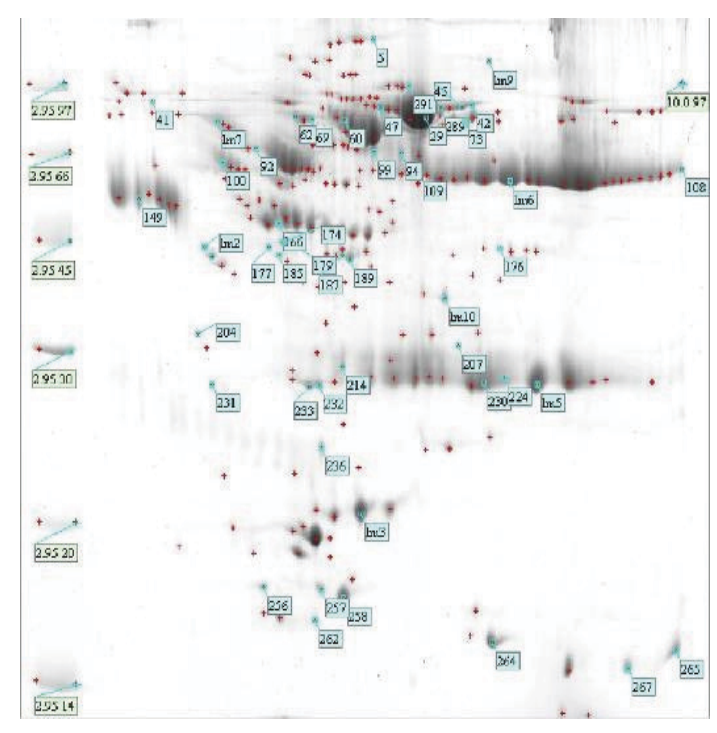

Fig. 5. The 2-DE map of samples in the MPE group. Note: $\mathrm{pH}$ range from 3 to 10 ; molecular weight from14-97 kD. 


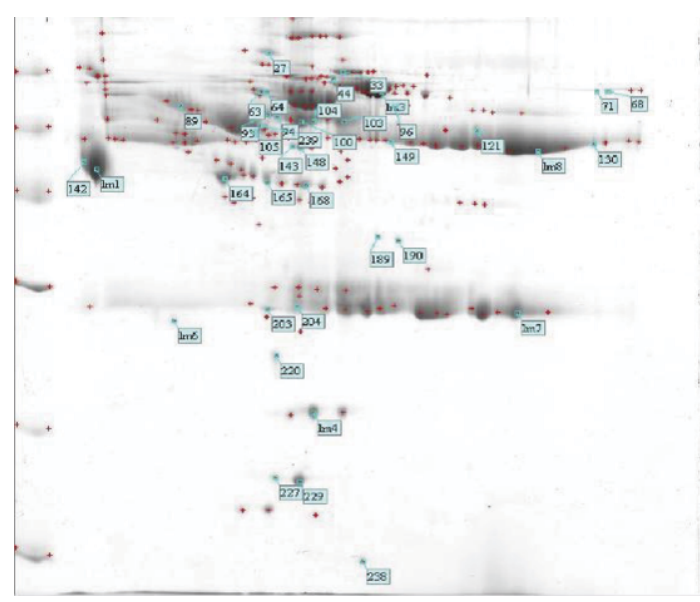

Fig. 6. The 2-DE map of samples in the TSPE group. Note: $\mathrm{pH}$ range from 3 to 10 ; molecular weight from14-97 kD.

Table 1

Comparison of 2-DE maps between TBPE and MPE groups

\begin{tabular}{lllllllll}
\hline ID & Mean $(100 \%)$ & M.S.D & Variation & GT & GC & GT/GC & PI & MW \\
\hline $\mathbf{4 1}$ & $\mathbf{0 . 3 8 0 6 5 7}$ & $\mathbf{0 . 3 5 8 8 3}$ & $\mathbf{0 . 9 4 2 6 5 9}$ & $\mathbf{0 . 7 3 9 4 8 7}$ & $\mathbf{0 . 0 2 1 8 2 7}$ & $\mathbf{3 3 . 8 7 9 3 1}$ & $\mathbf{3 . 8 1}$ & $\mathbf{8 8}$ \\
$\mathbf{7 3}$ & $\mathbf{0 . 0 5 5 2 2 4}$ & $\mathbf{0 . 0 5 4 5 5 8}$ & $\mathbf{0 . 9 8 7 9 3 6}$ & $\mathbf{6 . 6 6 E - 0 4}$ & $\mathbf{0 . 1 0 9 7 8 2}$ & $\mathbf{0 . 0 0 6 0 6 9}$ & $\mathbf{7 . 4 8}$ & $\mathbf{7 8}$ \\
149 & 1.86681 & 1.17258 & 0.62812 & 3.03939 & 0.694229 & 4.37808 & 3.68 & 54 \\
256 & 0.067776 & 0.041407 & 0.610936 & 0.026369 & 0.109183 & 0.241515 & 5.14 & 17 \\
257 & 0.110344 & 0.06922 & 0.627311 & 0.041124 & 0.179564 & 0.229021 & 5.79 & 17 \\
$\mathbf{2 5 8}$ & $\mathbf{0 . 5 0 9 8 6 1}$ & $\mathbf{0 . 2 1 1 3 8 7}$ & $\mathbf{0 . 4 1 4 5 9 7}$ & $\mathbf{0 . 2 9 8 4 7 4}$ & $\mathbf{0 . 7 2 1 2 4 8}$ & $\mathbf{0 . 4 1 3 8 3}$ & $\mathbf{6 . 0 6}$ & $\mathbf{1 7}$ \\
262 & 0.245015 & 0.16329 & 0.666449 & 0.408305 & 0.081725 & 4.996091 & 5.73 & 16 \\
265 & 0.206539 & 0.133275 & 0.645277 & 0.073264 & 0.339813 & 0.215601 & 9.94 & 15 \\
\hline
\end{tabular}

obtained the2-DE images of the three sample groups displayed in Figures 4-6. These images were compared in pairs, as shown in Tables 1-3. Out of the 24 proteins identified by mass spectrometry, eight protein points show significant differences between the TBPE and MPE groups, ten between the TBPE and TSPE groups and six between the MPE and TSPE groups. We established standards for choosing the differential protein points as follows: a) The grayscale of the differential points rises more than two times or drops more than two times; b) In combination with the images, the depth and size of the points demonstrate significant differences; and c) The protein points must fall in the $\mathrm{pH}$ range of 3-10. Table 4 shows the MALDI-TOF-MS of different proteins and the features of related structure proteins. We found 31 protein points with significant differences among the three groups; subsequently, we chose the 18 points relevant to TBPE and identified them, as displayed in Table 4. All PMFs were obtained using MALDI-TOF/TOF-MS AB4700 (Brooke Dalton company). Instrument settings were as follows: $20 \mathrm{Kv}$; positive ions; matrix with CCA; internal standard: degradation peak 2163.05 of the trypsin; external standard: mixture of seven kinds of standard peptides: Angiotesin II (m/z: 1046.54), Angiotesin I (m/z: 1296.68), Substance P (m/z: 1347.74), Bombesin (m/z: 1619.82), ACTH clip (1-17) (m/z: 2093.09), ACTH clip (18-39) ( m/z: 2465.20) and Somatostatin(28) (m/z: 3147.47).

Finally, we retrieved the data with Mascot software (http://www.matrixscience.co.uk) in the NCBI database.

Retrieval parameters were set as: 
Table 2

Comparison of 2-DE maps between TBPE and TSPE groups

\begin{tabular}{|c|c|c|c|c|c|c|c|c|}
\hline ID & Mean $(100 \%)$ & M.S.D & Variation & GT & GL & GT/GL & $\mathrm{PI}$ & $\mathrm{MW}$ \\
\hline 27 & 0.154229 & 0.09932 & 0.643979 & 0.054909 & 0.25355 & 0.21656 & 5.71 & 97 \\
\hline 100 & 0.810672 & 0.45439 & 0.56051 & 0.356282 & 1.26506 & 0.281632 & 6.03 & 69 \\
\hline 130 & 0.473542 & 0.247407 & 0.52246 & 0.720949 & 0.226135 & 3.188135 & 9.58 & 57 \\
\hline 148 & 0.180481 & 0.084436 & 0.467838 & 0.096045 & 0.264917 & 0.362548 & 6.02 & 57 \\
\hline 165 & 0.409901 & 0.251253 & 0.612961 & 0.661154 & 0.158648 & 4.167427 & 5.61 & 46 \\
\hline 189 & 0.056765 & 0.033249 & 0.58572 & 0.090014 & 0.023517 & 3.827654 & 6.94 & 35 \\
\hline 220 & 0.101447 & 0.059797 & 0.589446 & 0.041649 & 0.161244 & 0.2583 & 5.72 & 24 \\
\hline 239 & $512 \mathrm{E}-04$ & $3.33 \mathrm{E}-04$ & 0.650002 & $8.46 \mathrm{E}-04$ & $1.79 \mathrm{E}-04$ & 4.714327 & 5.52 & 64 \\
\hline
\end{tabular}

Table 3

Comparison of 2-DE maps between MPE and TSPE groups

\begin{tabular}{lllllllll}
\hline ID & Mean (100\%) & M.S.D & Variation & G L & GC & GL/GC & PI & MW \\
\hline 298 & 0.297259 & 0.218982 & 0.736672 & 0.516241 & 0.0782766 & 6.595087165 & 5.93 & 92 \\
361 & 0.866247 & 0.719752 & 0.830886 & 1.586 & 0.146495 & 10.82630807 & 6.11 & 66 \\
380 & 0.86316 & 0.39572 & 0.458455 & 0.46744 & 1.25888 & 0.371314184 & 6.92 & 59 \\
431 & 0.744976 & 0.272932 & 0.366363 & 0.472044 & 1.01791 & 0.463738444 & 5.52 & 48 \\
439 & 0.304812 & 0.1074 & 0.352347 & 0.197412 & 0.412212 & 0.478908911 & 6.34 & 45 \\
593 & 0.381374 & 0.381335 & 0.999899 & 0.762709 & $3.84 \mathrm{E}-05$ & 19874.27267 & 6.25 & 21 \\
\hline \multicolumn{7}{c}{ Note: GL: Gray scale of TSPE; GC: Gray scale of MPE; GT: Gray scale of TBPE. }
\end{tabular}

Table 4

Results of the MALDI-TOF-MS identification of the 18 points

\begin{tabular}{|c|c|c|c|c|c|c|}
\hline $\begin{array}{l}\text { Spot } \\
\text { No. }\end{array}$ & Accession No. & $\begin{array}{l}\text { Molecular mass } \\
\text { (MS/PI ) }\end{array}$ & $\begin{array}{l}\text { Sequence } \\
\text { Coverage }\end{array}$ & $\begin{array}{l}\text { Mascot } \\
\text { score }\end{array}$ & Protein name & $\begin{array}{l}\text { Raise or } \\
\text { drop }\end{array}$ \\
\hline 41 & gi $\mid 146386605$ & $43823 / 7.40$ & $32 \%$ & 95 & C1-inhibitor & $\downarrow$ \\
\hline 258 & gi|3891560 & $13783 / 5.35$ & $99 \%$ & 90 & TTR & $\uparrow$ \\
\hline 190 & gi 194375572 & $57477 / 6.12$ & $20 \%$ & 104 & Unnamed protein & $\downarrow$ \\
\hline 148 & gi| 237823916 & $46438 / 5.54$ & $30 \%$ & 72 & Human fibrinogen $\mathrm{C}$ chain & $\downarrow$ \\
\hline 130 & gi $\mid 20664303$ & $23503 / 7.16$ & $68 \%$ & 84 & Z34c-Fc & $\uparrow$ \\
\hline 27 & gi $\mid \mathbf{1 5 7 8 3 1 5 9 7}$ & $120009 / 5.41$ & $39 \%$ & 163 & $\mathbf{C P}$ & $\downarrow$ \\
\hline 73 & gi|317455056 & $71033 / 6.82$ & $53 \%$ & 165 & C3b & $\uparrow$ \\
\hline 165 & gi|3337390 & $38209 / 6.14$ & $31 \%$ & 67 & Haptoglobin & $\downarrow$ \\
\hline 204 & gi|90108664 & $28061 / 5.27$ & $74 \%$ & 145 & Hybrid protein & $\uparrow$ \\
\hline 220 & gi|33357204 & $21076 / 5.27$ & $77 \%$ & 70 & $\begin{array}{l}\text { Recombinant human serum } \\
\text { retinol-binding protein }\end{array}$ & $\uparrow$ \\
\hline 262 & gi|4503109 & $16204 / 4.95$ & $75 \%$ & 52 & $\begin{array}{l}\text { Precursor of cysteine } \\
\text { protease inhibitors-S }\end{array}$ & $\uparrow$ \\
\hline 257 & gi|56090473 & $21132 / 9.61$ & $24 \%$ & 47 & $\begin{array}{l}\text { Cancer / testis antigen } \\
\text { family A5 }\end{array}$ & $\downarrow$ \\
\hline 256 & gi|116283372 & $47047 / 7.53$ & $44 \%$ & 50 & CCDC67 protein & $\downarrow$ \\
\hline 239 & gi|31615935 & $23186 / 7.12$ & $41 \%$ & 65 & The IgA crystal structure & $\downarrow$ \\
\hline 189 & gi|33341706 & $46673 / 9.31$ & $28 \%$ & 58 & FP938 protein & $\downarrow$ \\
\hline 149 & gi|219519923 & $23532 / 4.93$ & $50 \%$ & 63 & serum mucin 1 & $\uparrow$ \\
\hline 265 & gi|154813201 & $36938 / 8.80$ & $18 \%$ & 46 & ALX homologous protein & $\downarrow$ \\
\hline 8 & gi|157831596 & $44226 / 5.37$ & $50 \%$ & 81 & Hybrid protein & $\uparrow$ \\
\hline
\end{tabular}

Note: The symbol $\uparrow$ indicates that the grayscale of the differential points rises at least two times more than the selected standard; the symbol indicates that the grayscale of the differential points drops at least two times more than the selected standard. 


\begin{tabular}{ll}
\hline Database & nrNCBI \\
Taxonomy & Homo sapiens \\
Enzyme & Trypsin \\
Allow up to & 1 \\
Variable modifications & Carbamidomethylation (C) \\
& Oxidization (M) \\
Peptide tol. + & $0.3 \mathrm{ppm}$ \\
\hline
\end{tabular}

Using PMFs, we can judge whether a protein is a differential protein that needs to be identified according to molecular weight, isoelectric point, matching number of peptides, sequence coverage rate and scores given by the software. Ultimately we obtained the 18 relevant structure proteins' peptides sequences and PMFs.

\subsection{Statistical analysis}

Post-hoc testing (Newman-Keuls) on the protein expression index of each point judged whether the differences are statistically significant. To determine whether the differential proteins can serve as symbol proteins, we used paired $t$-tests to determine their statistical significance $(\mathrm{P}<0.05)$.

\section{Results}

We found 24 differential protein points for which isoelectric points range from 3 to 10 and molecular weight ranges from 14-97 kD. Tables 1-3 display the specific results. The 188 different protein points between the TBPE and MPE groups, we chose the eight most significant points and identified them in the database: C1-INH (point 41), TTR (point 258), C3b (point 73), serum mucin 1 (point 149), CCDC67 protein (point 256), cancer/ testis antigen family A5 (point 257), precursor of cysteine protease inhibitors-S (point 262), and ALX homologous protein (point 265). Form the 154 different protein points between the TBPE and TSPE groups, we chose the ten most significant ones and identified them in the database: Ceruloplasmin (point 27), haptoglobin (point 165), hybrid protein (point 204), recombinant human serum retinol-binding protein (point 220), the IgA crystal structure (point 239), FP938 protein (point 189), hybrid protein (point 8), Z34c-Fc (point 130), human fibrinogen C chain (point 148), and unnamed protein (point 190). From the 161different protein points between the MPE and TSPE groups, we chose the 13 most significant ones and identified them in the database: D-dimer, fibrin B chain (point 439), family protein mixture (point 361), unnamed protein (point 6), $\beta$-globin (point 5), precursor of Apolipoprotein 1 (point 4), a chain of Apolipoprotein A1 (point 1), HP protein (point 431), unnamed protein (point 593), Fibrinogen fragment D (point 380), precursor of Apolipoprotein 1 (point 3), $\gamma$-fibrinogen (point 298), the IgA crystal structure (point 7), and the DNA binding protein of the Chromatin helicase (point 2). Out of the161 different protein points between MPE and TSPE, six failed to achieve significant differences. In total, we found 18 protein points with significant differences. We used the MALDI-TOF/TOF-MS to acquire the PMFs of the 18 protein points, which are shown in Table 4. We found additional significance in the comparison of C1-INH, TTR, C3b, CP, and Z34c-F; therefore, we made three two-dimensional gels of different pleural effusions, located the stable proteins (point 258, 41, 73, 27, 130) and analyzed them by gel-pro analyzer 4.0(Media Cybernetics) (see Figure 7). C1-INH shows a high expression in TBPE 


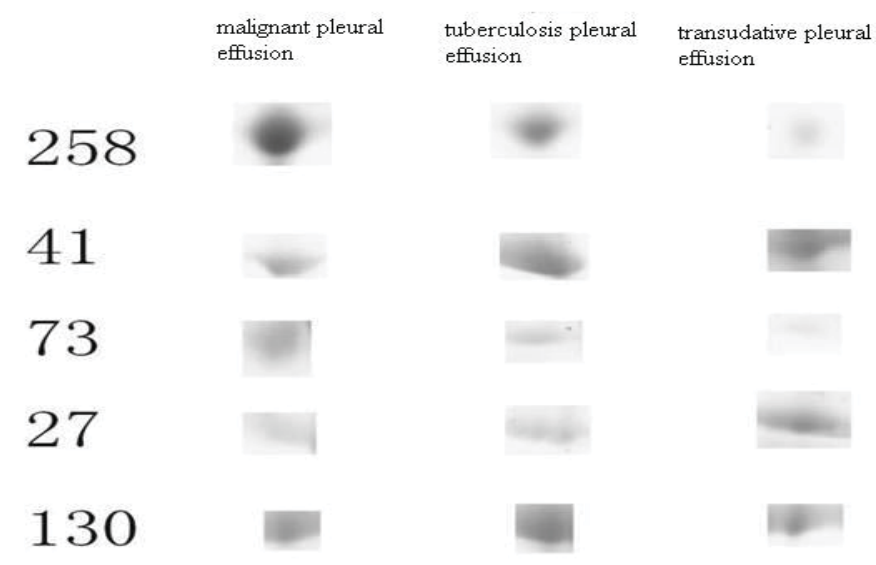

Fig. 7. Stable and variable characteristics of the three groups.

and a low expression in MPE while TTR and C3 show low expressions in TBPE and high expressions in MPE. Z34c- Fc and CP have a higher expression in TBPE than in TSPE. No characteristic protein was found in common between MPE and TSPE. We examined the differences in expression using paired $t$-tests, and the difference between them is significant $(\mathrm{P}<0.05)$.

\section{Discussion}

Progress in new diagnostic techniques for major disorders is generally measured not in years but in decades or centuries. Fortunately, in the last decades, remarkable progress has been made in the rapid and accurate diagnosis of tuberculosis pleural effusion. In an era of molecular biology, molecular methods present many advantages compared with more conventional diagnostic methods [6]. As the field of proteomics has developed, many studies have attempted to locate diagnostic proteins in pleural effusions. Several studies [7-9] showed that INF- $\gamma$ in pleural fluid may be the most sensitive and specific among other biological markers for tuberculous pleuritis. Furthermore, C-reactive protein (CRP) as a marker of inflammation has proven to be sensitive and specific for differentiating parapneumonic effusion from other etiologies of pleural effusion [10]. Another study [11] shows that a simple marker of elevated CRP level in pleural fluid may be helpful in discriminating between TBPE and MPE. In our study, we compared three different kinds of pleural effusions and found five proteins with significance for differentiating the three sample groups.

The complement system is thought to be triggered by a foreign pathogen, such as pyogenic bacteria or mycobacterium tuberculosis. C1-INH, an important member of the complement system, works in the early stage of the system's activation through a classic pathway. Huyen T.T. Tran confirmed the increased expression of $\mathrm{C} 1-\mathrm{INH}$ in monocytes of TB-associated immune reconstitution inflammatory syndrome (TB-IRIS) patients [12]. Similarly, a case report published in 2008 described an unusual association of disseminated tuberculosis with hereditary C1-INH deficiency (C1-INH D) in a Tunisian man [13]. These results match our finding of the high expression of C1-INH in TBPE.

Recently, research increasingly shows that the complement system plays an important triage role by differentiating between healthy host cells, microbial intruders, cellular debris and immune complexes [14]. C3b works through an alternative pathway to C1-INH and is essential for directing attacks on malignant cells and protecting healthy host cells [15]. Several studies focusing on MPE patients report 
elevated levels of the complement factors $\mathrm{C} 3$ and C4 in MPE [16, 17]. The present study found C3b levels to be higher in MPE and lower in TBPE, but the different amounts of C3b were shown in gray value. Quantitative two-dimensional gel proteomics should further explore this finding in the future.

Our study also found TTR levels to be higher in patients with MPE. Previous research has shown the TTR level to be upregulated in the sera of adenocarcinoma lung cancer patients using 2-DE and MALDI-TOF-MS peptide mass fingerprinting [18]. Another recently published Chinese population based study of patients with lung cancer found that both TTR levels in pleural effusion and pleural effusion to serum TTR ratios were higher compared with patients with noncancerous lung infections [19]. These results along with our findings indicate that TTR has the potential to contribute to differential diagnosis among different pleural effusions.

Copper is unique among the essential trace minerals in that it exists in the oxidation state and is highly redox active. Most hepatic copper is incorporated into the CP protein, which is subsequently secreted from hepatocytes into the blood [20]. One study compared different acute phase proteins and showed that $\mathrm{CP}$ is significantly increased compared to other proteins in exudative pleural effusion [21]. A recently published article focuses on the differential diagnosis between exudates and transudates an report both that $\mathrm{CP}$ levels are much higher in exudative pleural effusions and that the sensitivity and specificity of $\mathrm{CP}$ are much better than those of Light's criteria [22]. In the present study, we determined that $\mathrm{CP}$ levels are higher in TBPE than in TSPE. We conclude that CP plays an important role in the progress of exudative pleural effusion. However, the mechanism behind this relationship remains unknown and additional experimentation is required to elucidate the role of $\mathrm{CP}$ in pleural effusion.

$\mathrm{Z} 34 \mathrm{c}-\mathrm{Fc}$ is one of the derivatives of zinc finger proteins, which are now recognized to bind DNA, RNA, proteins, and lipid substrates. They play an important role ina wide variety of cellular processes including gene transcription, translation, mRNA trafficking, cytoskeleton organization, epithelial development, cell adhesion, protein folding, chromatin remodeling, and zinc sensing [23]. Ronald Benjamin found that HIV, Mycobacteria, and HIV-TB co-infections increased hZNF-134 expressions [24]. This suggests that zinc finger proteins may play an important role in the physiological and pathological processes of tuberculosis. However, to our knowledge, no research exists on the relationship between Z34c-Fc and pleural effusion. Our finding of the over-expression of Z34c-Fc in TBPE requires further confirmation.

\section{Study limitations}

This is a pilot study using 2-DE and MALDI-TOF-MS without quantitative two-dimensional gel proteomics. We were unable to verify the proteins; therefore, the different expressions of the proteins are presented as grey values. Further research should incorporate methods such as the western blot or ELISA to determine the acute amount of the different expressed proteins. The small size of samples and protein sensitivity limits the detection of low-content proteins that occupy a large proportion in a particular proteome. Proteins with high molecular weight (more than $200 \mathrm{kDa}$ ) and extremely acidic or alkaline proteins always get lost in electrophoresis, so the proteins separated by 2-DE are not all inclusive. On the other hand, the protein points on the electrophoresis glue can include more than one type of protein, and the various components of the mixture proteins in the same point may differ on six orders of magnitude or more. Sometimes, the same protein will form different points because of different modifications. All of these possibilities make comprehensive quantitative analysis of protein expression extremely complicated. Moreover, the 2-DE method itself is a labor-intensive technique 
with high technical requirements, making it difficult to automate. Thus, the great challenges of comprehensive proteome research lie in separating protein mixtures and obtaining enough information about the proteins for analysis. The nature and functions of the proteins in pleural effusions also require further researches. In following up on this study, we can increase the number of samples, verify the proteins using the western blot methods, identify the clinical differential proteins, and further advance the goals of our research.

\section{Conclusion}

C1-inhibitor (C1-INH), Transthyretin (TTR), Human Complement fragment 3b (C3b), Human Ceruloplasmin $(\mathrm{CP})$ and $\mathrm{Z34c}$ protein fragment $\mathrm{Fc}(\mathrm{Z34c}-\mathrm{Fc})$ may provide a new method for the differential diagnosis of varying pleural effusions. Further quantitative studies should explore cut off values for these proteins.

\section{References}

[1] W.H. Organization Global Tuberculosis Report, Geneva, Switzerland, 2014.

[2] K. Kranzer, Improving tuberculosis diagnostics and treatment, The Lancet 377 (2011), 1467-1468.

[3] S.A. Sahn, State of the art: The pleural, American Review of Respiratory Diseases 138 (1988), 184-234.

[4] A. Gopi, S.M. Madhavan, S.K. Sharma, et al., Diagnosis and treatment of tuberculous pleural effusion in 2006, Chest 131 (2007), 880-889.

[5] H.-G. Batz, M. Casenghi, G.S. Cooke, et al., New research and development strategy for tuberculosis diagnostics urgently needed, The Lancet Infectious Diseases 12 (2012), 584-585.

[6] V. Seetha, T.D. Balasingham, Irena Szpinda, A. Stephan, A. Frye and Tone Tønjum, Molecular diagnostics in tuberculosis: Basis and implications for therapy, Molecular Diagnosis \& Therapy 13 (2009), 137-151.

[7] A.H. Keisuke Aoe, Tomoyuki Murakami, Ryosuke Eda, Tadashi Maeda, Kazuro Sugi and Hiroyasu Takeyama, Diagnostic significance of interferon- $\gamma$ in tuberculous pleural effusions, Chest Journal 123 (2003), 740-744.

[8] C.-F. Wong, W.-W. Ew, S.K.-F. Leung, et al., Assay of pleural fluid interleukin-6, tumour necrosis factor-alpha and interferon-gamma in the diagnosis and outcome correlation of tuberculous effusion, Respiratory Medicine 97 (2003), 1289-1295.

[9] V. Villena, A. López-Encuentra, F. Pozo, et al., Interferon gamma levels in pleural fluid for the diagnosis of tuberculosis, The American Journal of Medicine 115 (2003), 365-370.

[10] M.X. Zou, R.R. Zhou, W.J. Wu, et al., The use of pleural fluid procalcitonin and c-reactive protein in the diagnosis of parapneumonic pleural effusions: A systemic review and meta-analysis, American Journal of Emergency Medicine 30 (2012), 1907-1914.

[11] A.K. Nitipatana Chierakul, Angkana Chaiprasert and Ronnachai Viriyataveekul, A simple c-reactive protein measurement for the differentiation between tuberculous and malignant pleural effusion, Respirology 9 (2004), 66-69.

[12] H.T. Tran, R. Van den Bergh, M.M. Loembe, et al., Modulation of the complement system in monocytes contributes to tuberculosis-associated immune reconstitution inflammatory syndrome, AIDS 27 (2013), 1725-1734.

[13] M. Kallel-Sellami, J. Blouin, R. Abdelmalek, et al., Disseminated tuberculosis in a patient with hereditary C1-inhibitor deficiency, Infection 36 (2008), 181-183.

[14] D. Ricklin, Manipulating the mediator: Modulation of the alternative complement pathway C3 convertase in health, disease and therapy, Immunobiology 217 (2012), 1057-1066.

[15] E.T. Sayegh, O. Bloch and A.T. Parsa, Complement anaphylatoxins as immune regulators in cancer, Cancer Medicine 3 (2014), 747-758.

[16] S.C.M. Alexandrakis, D. Kyriakou, D. Bouros, N. Xirouhaki, E.C.N. Siafakas and G.D. Eliopoulos, Diagnostic value of ferritin, haptoglobin, $\alpha 1$-antitrypsin, lactate dehydrogenase and complement factors $\mathrm{C} 3$ and $\mathrm{C} 4$ in pleural effusion differentiation, Respiratory Medicine 91 (1997), 517-523.

[17] M.G. Alexandrakis, D. Kyriakou, I.E. Koutroubakis, R. Alexandraki, I.G. Vlachonikolis and G.D. Eliopoulos, Assaying of tumor necrosis factor factors, and malignant serous effusions, American Journal of Clinical Oncology-Cancer Clinical Trials 24 (2001), 562-565. 
[18] M.J. Carla Mendes Maciel, Marcos Eduardo Machado Paschoal, Marcia Tie Kawamura, Ricardo Luiz Menezes Duarte MdGdCC and Gilberto Barbosa Domont, Differential proteomic serum pattern of low molecular weight proteins expressed by adenocarcinoma lung cancer patients, Journal of Experimental Therapeutics and Oncology 5 (2005), 31-38.

[19] H. Ding, J. Liu, R. Xue, et al., Transthyretin as a potential biomarker for the differential diagnosis between lung cancer and lung infection, Biomedical Reports 2 (2004), 765-769.

[20] S. Gulec and J.F. Collins, Molecular mediators governing iron-copper interactions, Annual Review of Nutrition 34 (2014), 95-116.

[21] M.S.C. Calikoğlu, A. Unlü, A. Kanik, L. Tamer and I. Calikoğlu, Use of acute phase proteins in pleural effusion discrimination, Tuberkuloz ve Toraks 52 (2004), 122-129.

[22] G.K. Shanthaveeranna, V.G. Thykadavil and G.A. D'Souza, Use of pleural fluid ceruloplasmin in the differentiation of exudative and transudative pleural effusion, Lung India 32 (2015), 11-15.

[23] J.H.L.B. Laity and P.E. Wright, Zinc finger proteins: New insights into structural and functional diversity, Current Opinion in Cell Biology 11 (2011), 39-46.

[24] A.B. Ronald Benjamin, Kannan Balakrishnan, Ramya Sivangala, Sumanlatha Gaddam and Sharmistha Banerjee, Mycobacterial and HIV infections up-regulated human zinc finger protein 134, a novel positive regulator of HIV-1 LTR activity and viral propagation, PLoS One 9 (2014), e104908. 